\title{
Social Computing Empowered Cloud Service on Quantitative Investment
}

\author{
Li Zhang ${ }^{1}$, Peng Chen, Jun'e Liu and Qian Li \\ ${ }^{1)}$ Software Engineering Center of Chinese Academy Science, Beijing, China (lzhang@ @sec.ac.cn)
}

\begin{abstract}
The quantitative investment achieves investment philosophy with the help from mathematical model, implements a trading strategy with program. The rapid development of the securities industry has changed the quality of the investor. Investment approach with a combination of means of information technology has changed the capital markets. In this paper, we propose a system of Cloud-based Quantitative Investment which comes from project about "Researching and Operating Securities industry Cloud". This project is composed of quantitative investment service strategy, service implement, service operate. Cloud technology forms social computing environment, provides powerful computing and storage capability, promote the market participation and the Innovative capacity of the financial institution.
\end{abstract}

Keywords - Quantitative investment, Cloud Computing, Crowedsourcing, Financial Product Design, Cloud Service Operation

\section{社会计算推动下的量化投资云服务}

\author{
张莉 $^{1}$ 陈鹏 刘军娥 李倩 \\ 1) 北京中科院软件中心有限公司, 北京, 中国
}

摘 要 量化投资是指以数理模型来实现投资理念, 通过程序来实现交易策略的一种投资方法。证券行业的快速发展使投资者基 础和素质发生了质的变化, 投资方法与信息手段的结合正在改变着证券市场的投资业态。本文介绍了国内量化投资的基本情况与市场 需求，分析了该领域相关的技术，结合与上海证券交易所合作的 “证券业云平台研发与运营” 课题，提出了基于云计算的量化投资金 融服务体系, 针对云计算模式下量化投资服务战略、服务实现与服务运营进行了全面阐述。作者认为, 云计算技术形成了社会化计算 模式，该模式为量化投资提供了强大的运算、存储能力，对推动市场参与度，提高金融机构创新能力具有非常重要的意义。

关键词 量化投资, 云计算, 众包, 金融产品设计, 云服务运营

1. 引言

量化投资是最近十年来在国际投资界兴起的一个新方 法, 发展势头迅猛, 和基本面分析、技术面分析并称为三 大主流方法。量化投资利用数学、统计学、信息技术等领 域知识对投资全过程进行管理, 简单地说, 就是基于大量 历史交易数据和金融数据, 用算法和模型对选股、择时、 资产组合、交易、风险控制和绩效考核等环节进行操作和 管理。

科技部科技支撑项目 “证券和金融产品交易综合服务平台研发及 应用示范” 项目编号 (2012BAH13F00), 子课题 “证券云研发与 运营” 课题编号 (2012BAH13F02) 支持

\section{2. 云计算环境下的量化投资服务战略}

在国内, 随着证券行业的快速发展，投资者基础和素 质发生了质的变化, 证券市场在经济发展的推动下酝酿着 新的发展机会, 量化基金产品数量逐年增加。截至 2012 年 9 月 14 日, 共有 22 只量化型券商理财产品（发行规模为 142.74 亿, 占券商集合理财产品的 4.2\%)、18 只量化基金 (发行规模为 281.7 亿，占全部基金发行额的 $1.06 \%$ )、40 只量化型阳光私募产品成立[1]。

随着中国期货公司、资产管理公司牌照的发放, 国债、 期货、沪深 300、ETF 的上市, 期货期权等投资环境的变 化, 量化投资技术在中国投资领域得到了日益广泛的应用, 展现了巨大的发展空间, 对国家金融体系建设, 金融安全 
防御和综合国际金融抗风险能力的提升发挥着越来越重要 的作用。

面对迅速发展的量化投资领域, 机构和中小投资者在 数据平台、研究平台、交易平台和管理平台方面的需求日 益增长:

- 策略编写代码工作量大、代码复杂;

- 策略验证需要完善的数据支持;

- 策略的计算和运行需要大量的计算;

- 策略风险分析需要完善的数据基础;

在市场中, 核心机构 ${ }^{1}$ 和中介机构 ${ }^{2}$ 提供量化投资金融服 务需要使用更复杂的交易基础设施, 依赖规模较大的 IT 团 队, 团队中通常包括操作系统专家、网络高手们、数据库 管理员、软件开发工程师, 而且电话和 IT 支持都需要覆盖 这些系统的前端到后端, 甚至全球。近年来, 多资产, 多 司法管辖区的投资战略需要满足严格的监管和客户期望。

IT 成本的增加在机构内部面临非常大的压力[2]。

云计算技术的发展和成熟能很好地解决上述问题, 通 过使计算分布在大量的分布式计算机上，形成的社会化计 算环境, 这些环境包括: 非核心业务数据的存储、基础设 施、常用基础计算软件服务, 它们为量化投资服务提供了 技术层面的有力支持，同时社会化计算模式为量化投资策 略的设计、验证和运行以及风险评估提供了市场，量化投 资领域新的业务形态在技术和市场的推动下逐渐清晰, 如 图 1 所示。

通过建立量化投资云平台，形成新的量化投资设计、 开发、销售模式。云平台提供的主动搜索、社会化计算能 力将投资策略研发中心与软件开发商、策略采购方链接在 一起, 采用众包方式, 即一个公司或机构把过去由员工执

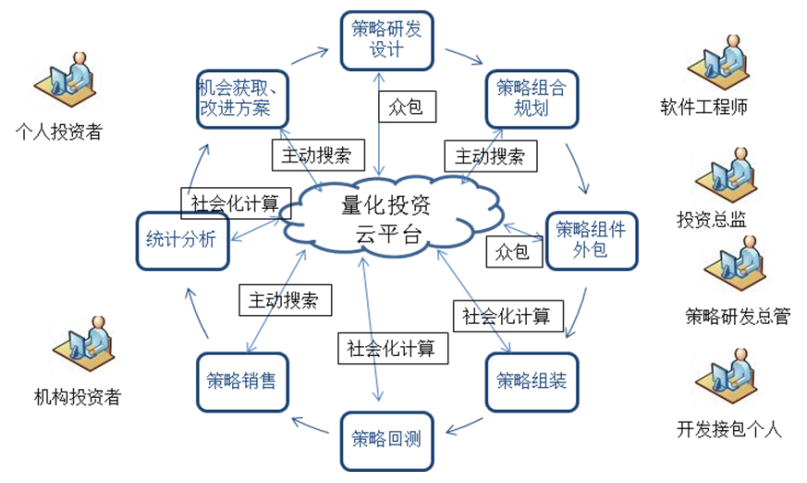

图 1 云计算环境下量化投资业态示意图

\footnotetext{
1 核心机构: 指中国资本市场核心机构包括沪深两市交易所、中 国期货交易所、中国证券登记结算中心、证券投资者保护基金、 期货保证金监控中心

2 中介机构: 指证券公司、期货公司、基金公司
}

行的工作任务, 以自由自愿的形式外包给非特定的（而 且通常是大型的）大众网络的做法, 将策略设计能力、 计算能力、软件开发能力引向投行、商业银行、卖方研 究机构、资产管理公司、公募及私募基金，从而提高金融 创新能力。

\section{3. 量化投资服务实现中的关键技术}

发展量化投资对资本市场是机遇，同时还面临一些困 难。除了众所周知的市场限制, 高质量的量化模型的设计 和开发需要投入较高的成本, 其中包括人力成本、数据成 本和 IT 系统成本, 如图 2 所示。

\section{1 智件}

所谓智件, 是指个人的经验、技能(诀空)、价值观等知 识[3]。投资领域经过两百多年的发展, 已经形成基本面分 析、技术分析、套利策略、趋势追踪、对冲交易、中性理 财等投资方法, 其中量化投资者更是需要具备数学、几何 学、物理学、心理学、仿生学等领域的知识来解释资产价 格的变化, 能运用从微分方程到数值方法, 从心理学理论 到人工智能等各种方法与工具, 寻找影响资产价格的各种 因素和规律。不仅如此, 从事量化投资还需要具备丰富的 操盘经验, 良好的投资直觉。业内针对这种情况开展了广 泛的理论探索和技术研究。这个过程中的关键环节是对投 资经验、投资思想甚至投资直觉这些投资智慧凝练成可复 用的成果理论，我们称之为投资智件。

目前在投资领域, 相当数量的模型都比诺贝尔经济学 奖级别的模型要复杂, 有的是模型看起来极为精简, 但是 训练模型的方法却极其复杂。对于相当多的模型算法, 使 用者是很难了解到算法发明者的全部意图和模型缺陷的, 甚至就连模型算法发明者自己，也有相当部分没有意识到 自己模型算法的缺陷。

\section{2 数据标准}

中国量化投资研究院报告称, 公募量化投资产品上半 年平均收益率为 $6.87 \%$, 高于整体公募基金的收益率。上 半年私募量化投资产品平均收益率是 $3.61 \%$, 券商理财量 投产品平均收益率仅为 $0.69 \%[4]$ 。, 可见量化投资的收益率 还是具有一定的吸引力, 但是由于大多数投资者能获得的 金融数据缺乏准确性, 加上金融衍生工具缺乏, 以及对高 频交易的限制等制约了量化投资的全面发展。

高质量的数据是做量化投资的基础, 国外在该领域已 有近 30 年的发展历史, 优质的数据仅仅是第一步, 审查和 过滤数据则是进一步保证量化模型质量的步骤。即使是最 
好的提供商的数据, 对冲基金公司也不能放松, 需要得有 自己的工具来检查数据。

如何提高各种金融数据的公信力和易用性既是业内的 关注焦点, 目前国内各大金融信息服务商都在该领域加强 投入, 推动包括: 盈利一致预期、全球财务数据、时间序 列估值在内的一系列 “云数据标准”。

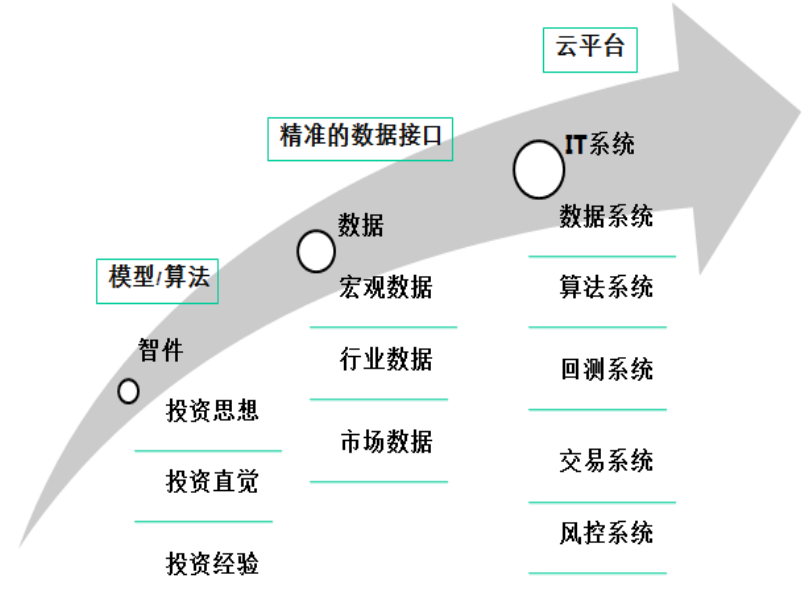

图 2 量化投资研究的关键领域

\section{3 量化投资工具}

量化投资量化和程序化的特点使得量化产品从设计、 回测、交易、风控以及监管等多个环节必须依赖软件工具。 这些工具需要具备灵活的系统配置, 以适应不同的市场和 金融衍生品; 需要安全开放的接口便于二次开发, 等等。 仅就风控系统而言, 实施量化交易的机构, 一年需要维护 和监管的模型数量少则几十, 多则成百上千。这里面存在 的最大难题在于, 交易策略、风险管理、产品设计、市场 营销等本身属于不同模型范畴, 各个类型下面又分很多子 类, 也是不同模型范畴, 比如交易策略, 基于不同假设、 背景、设计原理、采用算法、实现手段五花八门, 很多还 是黑箱算法, 模型代码的直接可解释程度很差。因此与量 化投资相关的投资工具种类很多, 使用难度随着技术发展 越发复杂。

按照数学模型的理念和对计算机技术的利用方式, 仅 与量化交易相关的交易方式可以进一步细分为自动化交易 (Automatic Trading)、量化投资 (Quantitative Investment)、 程序化交易（Program Trading）、算法交易（Algorithm Trading) 以及高频交易 (High Frequency Trading)。这五种 量化交易方式的侧重点各有不同, 工具也不同, 要求量化 投资工具从交易系统的行情和基础数据、交易和执行、策 略研发和运营三个主要方面既要做到大而全, 也要做到深 而精。这对目前大中型金融机构的 IT 以及实际运营部门是
很大的挑战, 同时也提供了发展机遇。

目前的量化投资工具随着资本市场、计算机技术的发 展以及投资者素质的发展, 在开发语言、技术架构、系统 架构、策略模型方面有了很大的提高和完善。除了支持复 杂脚本语言实现策略逻辑外, 要支持直接使用 C++、JAVA 等开发语言实现复杂的策略逻辑; 一般为了追求执行效率, 不采用界面显示图表, 而采用多进程、多线程方式进行自 动化交易、程序化交易、算法交易, 甚至为了追求极致, 使用硬件技术进行高频交易等量化交易方式; 技术架构是 使用服务器执行策略的架构, 行情使用转发路径最少的极 速、深度行情, 交易通道采用专用、直连的交易通道进行 交易。行情和交易的延时都要求尽可能最低。在系统架构 上严格区分策略研发和策略运营执行两个阶段。对于策略 研发阶段, 需要多品种、多周期、多账户、多交易市场、 多策略、复杂金融工程包的支持, 以实现复杂的策略逻辑; 对于策略运营执行阶段, 系统架构要保证各种风控、应急 处理、交易方式和策略的平稳有效执行。系统的实现流程 除了满足交易本身的要求外, 还要满足机构本身的业务流 程和规范, 以及监管层的要求。

\section{4. 量化投资云平台的营造}

云计算有各种定义, 它并不是一个单纯的产品, 也不 是一项全新的技术, 而是一种产生和获取计算能力的新的 方式。它的出现对 IT 的应用和部署模式以及商业模式, 都 产生了极大的影响。量化投资云平台[5]在结合不同资源: 如网络基础设施、服务器、存储、软件、服务后会衍生出 不同的量化投资领域的商业模式, 形成该领域全新的业态。 仅从计算机软硬件厂商的角度考虑, 在新业态下可开展的 商业模式包括:

\section{第一种商业模式是构建及运营与量化投资相关的共有} 云平台, 向个人投资者和机构投资者提供量化投资相关的 云服务。例如, 向最终使用者提供的 Online services, 满足 投资者量化投资相关的 IT 基础设施, 满足其跨平台、跨地 区, 以及安全、可靠的需求, 除此之外还提供相关的计算、 存储能力, 私有量化云平台通常采用的技术架构是使用服 务器执行策略的架构, 行情使用转发路径最少的极速、深 度行情, 交易通道采用专用、直连的交易通道进行交易。 行情和交易的延时都要求尽可能最低。

第二种是 ISV/SI 等各种合作伙伴基于量化云平台开 发算法、策略以及风控、资产管理等各种云计算应用, 并 在云平台上销售这些应用, 为最终使用者提供数据、算法、 应用软件等服务。云计算的广泛应用, 将从根本上改变信 息获取和知识传播的方式, 促进基础设施运营、软件等信 
息产业向服务化转型, 催生跨行业融合的新型服务业态。 2005 年 7 月中国科学院研究生院管理学院提出了威客理 论[6], 其核心思想是: 人的知识, 智慧, 经验, 技能通过 互联网转换成实际收益的互联网新模式。主要应用包括解 决科学, 技术, 工作, 生活, 学习等领域的问题。人类的 知识和智慧将会因为互联网而被无限放大和传播, 并创造 出令人惊讶的社会财富。

量化投资云平台提供的 PAAS 服务, 将为致力于量化 投资领域的研究人员、技术开发人员提供施展才华的平台, 他们的投资智慧将直接对接投资市场。

第三种大的机构投资者可以选择量化云平台构建自己 的私有云平台, 量化投资云平台提供包括产品、技术、平 台和运维管理在内的全面支持, 这是客户自建的私有云的 运营模式。该模式下除了提供网络、硬件、计算和存储能 力的设计方案, 还需要为机构投资者提供包括前端和后端 的完整解决方案。前端方案包括: 在网上交易行情、交易 功能的基础上扩展, 增加综合服务模块。利用对投资者的 分级分类, 为投资者提供差异化服务, 包括通道的差异化、 资讯信息差异化、行情数据差异化、交易工具差异化、个 性化投资分析、资产组合分析等。并且整合内容管理系统 和即时通讯等多种服务手段, 为用户提供丰富的内容资讯, 为投资者提供在线服务和和信息主动实时推送服务。后端 方案即机构服务人员使用的管理平台, 面向系统管理员、 研究中心、数据采编等人员, 提供策略回测、历史数据回 放、信息发布功能以及证券高端数据、策略数据、交易数 据统计等功能, 是机构为投资者提供服务和生产的工作平 台。

\section{5. 结论与展望}

量化投资经过几十年的发展, 在全球资本市场动荡不 定, 规律难循的背景下, 被证明是最好的投资方法之一,
已逐步成为全球金融机构的主流投资方法，但在中国量化 投资还是一种新兴技术, 处于萌芽和发展阶段。本文结合 “证券业云平台研发与运营” 课题, 分析了云计算环境下, 量化投资领域服务将会形成的服务战略和服务模式, 新的 信息技术的应用将会给我国的资本市场带来新的机会, 并 形成新的产业形态。

但目前而言, 这种模式的推广还需要市场的认可过程。 首先, 人们对于“云计算”的安全性和可靠性仍心存疑虑。 毕竟, 投资者更愿意把核心的机密以及相关的数据和计算 过程放在自己手里。

另外, “云计算”可能会出现各种不同的标准, 无论是 管理还是技术标准, 很多都在讨论完善中。

然而, 云计算技术在证券行业的应用已经得到广泛的 认可, 在行业监管机构、核心机构的推动下, 证券云平台 基础设施逐渐完善, 基于云服务的交易所接入规范、应用 开发规范正在制定中。随着行业中金融衍生品的不断推出, 相信量化投资云服务将成为投资领域不可或缺的重要工具 和服务手段。

\section{参考文献(References)}

[1] Wind Information Co., "Wind Info", 2012.

[2] Sebastien Jaouen, "Why should a trading firm try to be an IT firm?" 2013.

[3] Liu Qianhua and JiangXianli, "Chinese Government Management Innovation-Decision volume", China Social Sciences Press, 2004, pp127.

[4] Chinese Academy of quantitative investment, "Chinese quantitative investment industry Semi-annual report", 2012.

[5] Microsoft China, "Microsoft's cloud computing strategy for China: Three different operating modes", 2010.

[6] BBS of School of Management, UCAS, 2005. 